\title{
VI. Diffusion of gases as an irreversible process
}

\section{S.H. Burbury F.R.S.}

To cite this article: S.H. Burbury F.R.S. (1907) VI. Diffusion of gases as an irreversible process, Philosophical Magazine Series 6, 14:79, 122-126, DOI: 10.1080/14786440709463664

To link to this article: http://dx.doi.org/10.1080/14786440709463664

$$
\text { 册 Published online: } 16 \text { Apr } 2009 .
$$

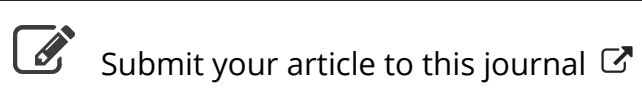

\footnotetext{
Џll Article views: 3
}

Q View related articles $\sqsubset$ 
As an example, consider the question of the stability of a symmetrical top spinning in small oscillation about the vertical when acted on by a periodic force through a point in the axis. If the force is vertical the equations of motion are :

$$
\begin{aligned}
-\mathrm{A} \ddot{\eta}+\mathrm{C} m \dot{\xi}+\mathrm{M} g h(1+\alpha \cos n t) \eta & =0, \\
\mathrm{~A} \ddot{\xi}+\mathrm{C} m \dot{\eta}-\mathrm{M} g h(1+\alpha \cos n t) \xi & =0,
\end{aligned}
$$

so that the effect of the applied force, to this degree of approximation, is to produce a variation in the spring alone; hence a comparatively large oscillation is generated if $n$ lies near to a certain value in the vicinity of $2 \mu_{1} / r$ or $2 \mu_{2} / r$, where $\mu_{1}$ and $\mu_{2}$ are the natural frequencies and $r$ any integer. If, on the other hand, the force is horizontal the spring is not affected, and there is instability only when $q=\mu_{1}$ or $\mu_{2}$. When the horizontal and vertical forces act together,

$$
\begin{aligned}
-\mathrm{A} \ddot{\eta}+\mathrm{C} m \dot{\xi}+\mathrm{M} g h(1+\alpha \cos n t) \eta=\mathrm{M} g h a \sin (q t+\epsilon), \\
\mathrm{A} \ddot{\xi}+\mathrm{C} m \dot{\eta}-\mathrm{M} g h(1+\alpha \cos n t) \xi=0 ;
\end{aligned}
$$

so that the joint action produces a large effect when $q=|c \pm r n|$, where $c$ represents either of the frequencies under the vertical force alone.

The spring and direct disturbances may be produced by giving the point of suspension periodic motions in the vertical and horizontal directions respectively; and for readily appreciable magnification it would be necessary to have the vertical vibration of large amplitude.

VI. Diffusion of Gases as an Irreversible Process. By S. H. Burbury, F.K.S.*

DRofessor G. H. Bryan in his recent work on Thermodynamics (Leipzig, Teubner \& Co.) states (p. 125), "when two gases at equal pressure and temperature mix by diffusion, the loss of available energy and consequent gain of entropy is the same as would occur if each component were to expand by rushing into vacuum till it occupied the same volume as the mixture." This statement appears to present some difficulties.

If in a cylinder of volume $2 v$, one half on one side of the piston is occupied by oxygen at given pressure and temperature, and the other half is vacuum, we have in that arrangement available energy. We might, by placing the axis of the cylinder vertical with the oxygen at the bottom,

\footnotetext{
* Communicated by the Author.
} 
cause the oxygen by its expansion to raise a weight, and so convert part of the energy of the oxygen into mechanical work $W$. If the gas is allowed to escape into the vacuum, no such mechanical work is done, or thereafter can be done, by the arrangement. We have lost an amount $W$ of available energy.

If instead of vacuum, the upper half of the cylinder be filled with oxygen at the same pressure and temperature as in the lower half, no mechanical work can be done by that arrangement. And therefore none can be lost by allowing the two volumes of oxygen to mix by diffusion. In fact if they do mix, the whole system remains in the same physical state, and therefore, by Art. 86 of Bryan's work, no entropy has been gained, nor available energy lost.

If the second half of the cylinder instead of with oxygen is filled with nitrogen at the same pressure and temperature, there is according to Bryan a loss of available energy for each component. But it is no more possible to use the oxygen-nitrogen arrangement for the practical purpose of doing work than to use the oxygen-oxygen arrangement. Where then is the loss of available energy when the oxygen and nitrogen are allowed to mix by diffusion?

The explanation is probably as follows :-Bryan asserts (p. 123), and he is a very high authority, (1) that when two gases mix by diffusion the process is an irreversible one. And I understand him to imply (2) that every irreversible process is attended by a loss of available energy. Both these positions (1) and (2) seem to be disputable, even against so high an autbority.

First as to the diffusion of gases being an irreversible process. We know by familiar experience, that if two gases mixed in different proportions but at the same pressure and temperature are separated by a partition, then, when we remove the partition, the gases begin to mix. That experience bowever, so far as regards the initial stages of the process, is as consistent with the diffusion being a cyclic-i.e. reversible - process as with its being an irreversible one. For if there be a cycle, it may be described in either of two directions, $\mathrm{ABC}$ or $\mathrm{CBA}$, one of which, in the given initial state of the system, is towards, and the other from, more uniform mixture. By removing the partition we determine the direction to be towards more uniform mixture. For let the gases be oxygen and nitrogen in a cylinder whose axis shall be that of $x$, and let the partition be at right angles to it, the oxygen being say at the left. Before the partition is removed, oxygen molecules striking it with velocity $u$ parallel to $x$ bave that 
velocity reversed, and owing to these reversals the mean $x$ velocity of all the oxygen molecules is zero. A short time after removal of the partition, those oxygen molecules which, but for the removal, would have had their $x$ velocities $u$ changed to $-u$, will still retain their original $x$ velocity $u$. So by removing the partition we have in effect given to the oxygen gas a mean momentum towards the (originally) nitrogen half of the cylinder, and to the nitrogen gas an equal mean momentum towards the oxygen half.

We thus determine the direction in which the cycle, if there be a cycle, is described.

In the limiting case of molecules having infinitely small diameters, so that collisions will not occur, it is evident that the motion must be cyclic. But it will be argued that the cyclic motion is destroyed by the collisions which actually occur. Before considering the general effect of this, we may note the application of it to the inference (2), which I understand Bryan to draw, namely, that the diffusion process, if irreversible, must on that account be attended by gain of entropy. May we not reason thus: The gain of entropy due to all the collisions is the sum of the gains of entropy due to the collisions separately. Therefore it is zero, because each separate collision is a reversible process attended by no gain of entropy? Is not the implied assumption that if the diffusion is an irreversible process, it must on that account involve gain of entropy, erroneous?

As to the general question of irreversibility, let the two diffusing gases be equal in quantity, and both at the same pressure and temperature. And let their respective densities at any point at any instant be denoted by $\rho$ and $\rho^{\prime}$. Let

$$
\mathrm{K}=\frac{1}{2} \int\left(\rho-\rho^{\prime}\right)^{2} d \tau,
$$

the integration being throughout the containing vessel. Then also

$$
\frac{d K}{d t}=\int\left(\rho-\rho^{\prime}\right) \frac{d \overline{\rho-\rho^{\prime}}}{d t} d \tau .
$$

It can then be proved on practically the same assumptions as those which are necessary in the proof of Boltzmann's $H$ theorem, though not by identically the same method, that $\frac{d \mathrm{~K}}{d t}$ is on average negative, so that $\mathrm{K}$ diminishes until it acquires its minimum value zero when $\rho=\rho^{\prime}$ everywhere.

The necessary assumptions are, in Boltzmann's language, "dass die Bewegung moleculär ungeordnet ist und für alle Folgezeit bleibt." I have tried to give a definition of the 
expression "moleculär ungeordnet," namely this :--The chance that any molecule at any instant shall have assigned velocities is independent of the relocities and positions of all the other molecules for the time being. This I cal! condition A. That, if it be accepted, is, so long as it remains true in fact, a sufficient foundation for the proof that $\frac{d \mathrm{H}}{d t}$, or $\frac{d \mathrm{~K}}{d t}$, is on average negative. But the state of things thus assumed to exist cannot possibly continue to exist indefinitely in a system completely isolated, that is completely protected from external disturbance. With my definition, therefore, the proof fails altogether that $H$ or $K$ continues irreversibly to diminish if the system be isolated.

If my definition be not accepted, will anybody make a better one? I think if he does he will tind that "moleculïr ungeordnet" represents a state of things which, like my condition A, cannot possibly continue to exist indefinitely in the isolated system.

The Loschmidt objection, that if you were to reverse all the velocities, the system would retrace its course, applies only to the isolated system, and as applied to the isolated system is in my opinion a conclusive objection to the theorem that the diminution of $\mathrm{H}$ or $\mathrm{K}$ is in that system irreversible.

The defence of the theorem which has met with wide acceptance, is that the reversed motion, although possible, is infinitely improbable compared with the direct motion. But if Maxwell's law prevails-and it cannot be denied by advocates of the $\mathrm{H}$ theorem-any set of velocities and the reversed set are equally probable.

If on the other hand the system be not isolated, but subject to disturbances, Loschmidt's objection is not true in fact. For on reversal of the velocities, the system would not retrace its course, beyond the point at which the last disturbance occurred. It may therefore be true that if disturbances are always occurring, e.g. if you keep stirring the mixture, $\mathrm{H}$ or $\mathrm{K}$ will go on diminishing to minimum.

I venture to suggest the following as the true theory. The motion Is in theory cyclic-i.e. reversible-in both cases, the $\mathbf{H}$ theorem and the diffusion-that is it is cyclic provided that the system be completely isolated. But that condition of complete isolation is impossible to realize in nature. How for instance can you prevent the gas in a closed vessel from being affected by vibrations coming from the surrounding medium? That is a sufficient reason why we can never expect the cyclic motion of two diffusing gases to become 
apparent. It is of no avail to say that the disturbances from without are very small, because a very small impulse may change the direction, and because the disturbances continue acting for an immense time. In the $\mathrm{H}$ theorem, when $\mathrm{H}$ is near its minimum, say $\mathrm{H}=\mathrm{H}_{0}+x$, where $x$ is very small, $\frac{d \mathrm{H}}{d t}$ is a small quantity of the second order, compared with $x$; so that in the cycle, if there be a cycle, $\mathrm{H}$ will remain near its minimum for very long periods of time. The same is true of $\mathrm{K}$ and $\frac{d \mathrm{~K}}{d t}$ in the diffusion theory.

The effect of disturbances coming from without is, as I pointed out in 'Nature' for November 1894, always to maintain or to renew pro tanto the state-_"moleculär ungeordnet," whatever that may mean-which is assumed to exist at every instant, as the necessary basis of proof that $\frac{d \mathrm{H}}{d t}$, or $\frac{d \mathrm{~K}}{d t}$, is negative. They must therefore be sufficient to destroy the possible cyclic motion. In that sense the diffusion of gases will, in any experiments that can practically be made, be found to be irreversible.

VII. Observations on the Electric Are. $B y$ Walter L. Upson, E.E., M.S., Princeton*.

[Plates VI. \& VII.]

\begin{abstract}
A LTHOUGH numerous investigators have studied the phenomena of the electric arc in air, between carbon terminals, and also between metal terminals, yet the use of the electric arc in the production of electric oscillations raises some new questions with regard to it.

While I was working in the Pender Electrical Laboratory of University College, London, during the present session, Prof. J. A. Fleming, F.R.S., suggested therefore to me that it would be interesting to make a further examination of the arc between metal and carbon terminals, in air and hydrogen.

I am indebted to him for the facilities for carrying out the work, and also for many suggestions during its progress. The following apparatus was first constructed with the kind assistance of Prof. W. C. Clinton, B.Sc.
\end{abstract}

* Communicated by the Physical Society : read June 14, 1907. 